OPEN ACCESS

Edited by:

Juliana L. Matos,

University of California, Berkeley,

United States

Reviewed by:

Yanpeng Wang,

Institute of Genetics and

Developmental Biology (CAS), China

*Correspondence:

Tedrick Thomas Salim Lew

tedrick@nus.edu.sg

orcid.org/0000-0002-4815-9921

Specialty section: This article was submitted to Genome Editing in Plants, a section of the journal

Frontiers in Genome Editing

Received: 31 December 2021 Accepted: 15 February 2022

Published: 07 March 2022

Citation:

Sharma $P$ and Lew TTS (2022) Principles of Nanoparticle Design for

Genome Editing in Plants.

Front. Genome Ed. 4:846624. doi: 10.3389/fgeed.2022.846624

\section{Principles of Nanoparticle Design for Genome Editing in Plants}

\author{
Pushkal Sharma ${ }^{1}$ and Tedrick Thomas Salim Lew ${ }^{2,3 *}$ \\ ${ }^{1}$ Department of Chemical Engineering, Massachusetts Institute of Technology, Cambridge, MA, United States, ${ }^{2}$ Department of \\ Chemical and Biomolecular Engineering, National University of Singapore, Singapore, Singapore, ${ }^{3}$ Institute of Materials Research \\ and Engineering, Agency for Science, Technology and Research (A*STAR), Singapore, Singapore
}

Precise plant genome editing technologies have provided new opportunities to accelerate crop improvement and develop more sustainable agricultural systems. In particular, the prokaryote-derived CRISPR platforms allow precise manipulation of the crop genome, enabling the generation of high-yielding and stress-tolerant crop varieties. Nanotechnology has the potential to catalyze the development of a novel molecular toolbox even further by introducing the possibility of a rapid, universal delivery method to edit the plant genome in a species-independent manner. In this Perspective, we highlight how nanoparticles can help unlock the full potential of CRISPR/Cas technology in targeted manipulation of the plant genome to improve agricultural output. We discuss current challenges hampering progress in nanoparticle-enabled plant gene-editing research and application in the field, and highlight how rational nanoparticle design can overcome them. Finally, we examine the implications of the regulatory frameworks and social acceptance for the future of nano-enabled precision breeding in the developing world.

Keywords: nanotechnology, gene editing, precision breeding, nanoparticles, CRISPR

\section{INTRODUCTION}

The Green Revolution in the 1960s enabled a steep rise in food security via the development of hybrid crops, fertilizers, and institutional mechanisms, benefitting many regions of the world by reducing malnourishment and poverty (Bailey-Serres et al., 2019). However, traditional cross-breeding and mutagenesis to generate desired traits are time-consuming and untargeted, and the crop yield improvement enabled by the Green Revolution has been steadily declining (Tilman et al., 2011; Chen et al., 2019). As a result, the current crop yields are estimated to fall short of the world's projected population demand by 2050 (Ray et al., 2013). This is exacerbated by ever-increasing abiotic and biotic stresses, limited genetic variation, and increasing resource costs (Bailey-Serres et al., 2019). Fortunately, with improvement in our understanding of underlying metabolic and protein interaction networks in plants, complemented by emerging genetic engineering technologies, it is possible to manipulate plant traits at the genomic level to help address these challenges. Recent progress in transgenic research has led to significant advances in engineering crops for improved yield and stress tolerance. Genome-wide association mapping has shown that single-nucleotide polymorphisms are enough to generate agriculturally-important trait variation in crop plants (Zhao et al., 2011; Hu et al., 2015; Bharat et al., 2020). Pathogen-recognizing receptor genes have been transferred between unrelated plant lineages to confer immunity against pathogenic strains (Boutrot and Zipfel, 2017; Koller et al., 2019), while the introduction of transcription factors from wetland species into staple crops has enabled repression of specific gene clusters to confer submergence tolerance without any yield penalty (Voesenek and Bailey-Serres, 2015; Dar et al., 2018). Plant 
biomass production has been increased considerably by modulating the chromatin accessibility by demethylase overexpression increasing crop yields (Yu et al., 2021), and manipulation of non-photochemical quenching-related enzymes helped tobacco plants in adjusting to fluctuating light conditions (Kromdijk et al., 2016). The ability to engineer gene expression also enables other applications such as the production of therapeutic compounds at scale (Fausther-Bovendo and Kobinger, 2021), rapid production of vaccines with increased immunogenicity (Fausther-Bovendo and Kobinger, 2021), removal of unwanted metabolites (Padmaja, 1995), or largescale knockout screens to probe unknown biological pathways (Gaillochet et al., 2020).

Rapid progress in genome editing technologies offers new opportunities to alter plant genome with nucleotide-scale precision for crop improvement. Base editing enzymes can perform point mutagenesis and have been used to generate herbicide-resistant crops (Shimatani et al., 2017; Tian et al., 2018; Zhang et al., 2019a; Bharat et al., 2020). The introduction of a single indel has been used to generate splice variants of the host plant translation factor disrupting its interactions with a viral protein which helped reduce the infection titer under field conditions (Gomez et al., 2019). While sequence-specific nucleases, such as zinc-finger nucleases and transcription activator-like effector nucleases (Wright et al., 2005; Christian et al., 2010), have been used for targeted editing of the plant genome, their application is limited due to the construction complexity of such large nucleases. CRISPR-Cas (clustered regularly interspaced short palindromic repeats/CRISPR-associated protein) is an alternative genome editing technology that relies on DNA-RNA binding for sequence-specific cleavage, offering design simplicity and ease of use at minimal cost (Mali et al., 2013; Koonin et al., 2017). The CRISPR-Cas toolbox is increasingly used to perform such genetic manipulation in plants, enabling gene knockout, base editing, organelle genome editing, and transcriptional regulation precisely in a targeted manner (Zhang et al., 2019b). As alluded earlier, non-native genes and accompanying desirable traits can be introduced into a wide range of plant species using transformation protocols where DNA is delivered into plant cells by either a particle gun or using plant-infecting soil bacterium Agrobacterium tumefaciens. However, these conventional methods insert varying copies of DNA at random locations in the host plant genome, leading to a low field performance (Meyer, 1995; Day et al., 2000; Dong and Ronald, 2021). Therefore these methods are usually supplemented by time-consuming regeneration of hundreds of independently transformation plant cells to screen for those with single-copy insertions and optimal phenotype (Mumm and Walters\&, 2001). On the other hand, CRISPR-Cas platform has been successfully used for targeted knock-in of markerfree DNA into specific endogenous genomic sites for optimized expression due to the high specificity of Cas nucleases and low off-target base-pairing rates of guide RNAs (Schiml et al., 2014; Zhao et al., 2016). Owing to their unparalleled ability to induce precise nucleotide changes, CRISPR-Cas systems have emerged as a powerful tool to improve crop yields and stress

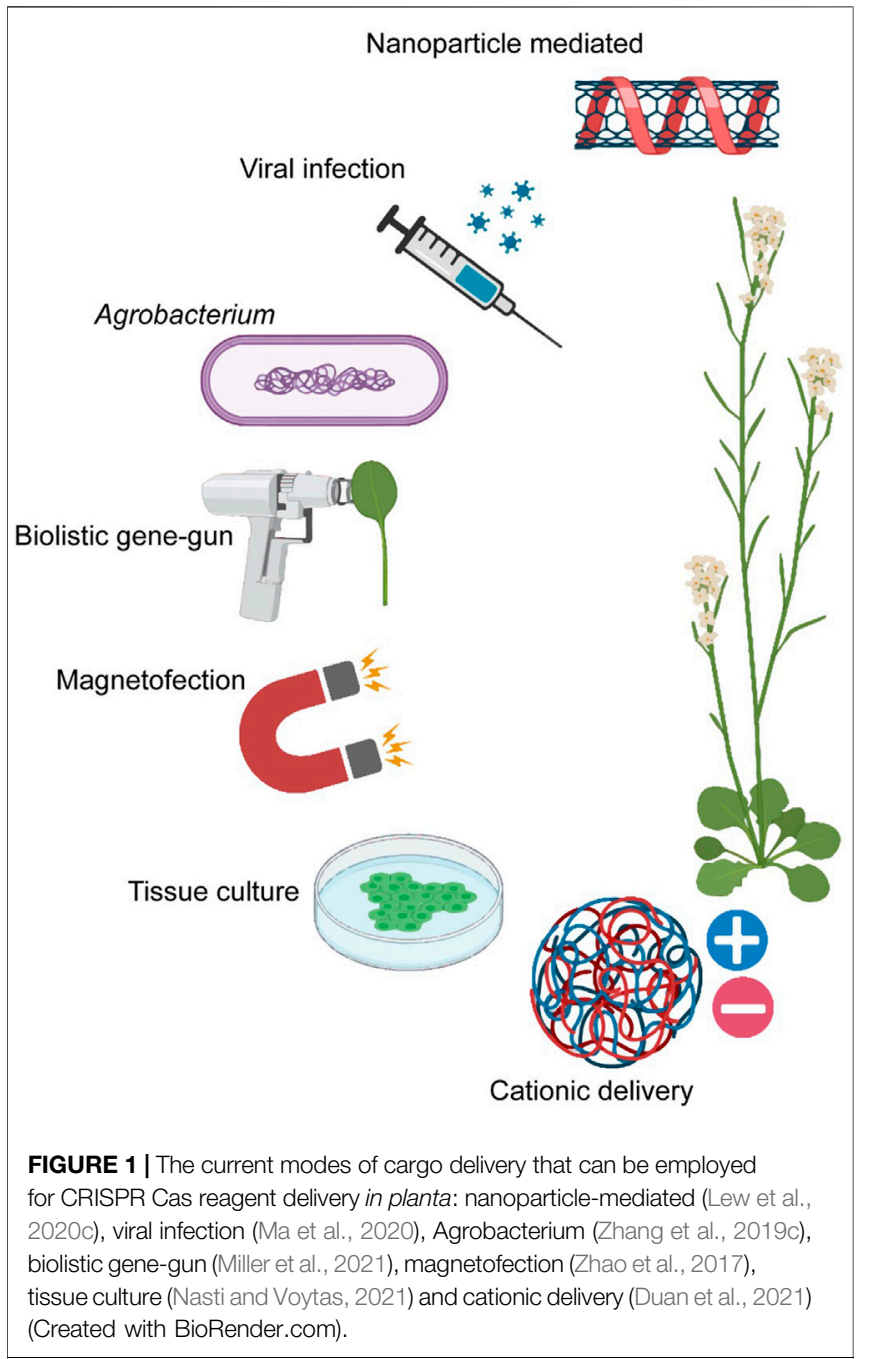

resistance. For example, disrupting Gn1a, DEP1, and GS3 genes in rice led to larger grain size and higher yield (Li et al., 2016), targeted mutations in the $M L O$ allele produced bread wheat resistant to powdery mildew (Wang et al., 2014), and editing the promoter region of host sucrose transporter genes generated rice lines with broad-spectrum resistance against bacterial blight (Oliva et al., 2019). By inducing targeted nucleotide variations to achieve the desired output, CRISPR has the potential to accelerate the transition from conventional cross-breeding to precision breeding for crop improvement.

An essential requisite for applying CRISPR technologies in agriculture is a robust and efficient way of delivering CRISPR reagents into plant cells. They are generally delivered into plant cells as plasmids or RNA-protein complexes by particle bombardment (Klein et al., 1992), Agrobacterium-mediated delivery (Zhang et al., 2019c), cationic delivery (Duan et al., 2021), or viral infection (Ma et al., 2020) (Figure 1). These conventional delivery systems are limiting in many ways. Agrobacterium or viral vectors apply only to a narrow range of species due to host-range constraints, and their efficiency in transformation efficiency is significantly affected by the plant 
genotype (Chen et al., 2019). Furthermore, Agrobacteriummediated delivery has yielded low transformation efficiencies (particularly in monocots) and is limited in modulating the amount of delivered donor templates which affects the insertion efficiency and frequency of unintended sequence disruptions (Mao et al., 2019). Alongside, it leads to random genomic integrations via nonhomologous recombination, which may disrupt essential genes $(\mathrm{SB}, 2017)$ and trigger regulatory concerns due to potential off-target mutations (Jones, 2015). Chemical methods such as cationic polymers are typically used to target protoplasts and thus require the establishment of suspension cells, protoplast isolation, and species-dependent regeneration protocols (Sandhya et al., 2020). Biolistic genegun or electroporation delivery is based on the mechanical rupturing of the target tissue by pressurized helium gas but is limited by tissue damage, nonspecific subcellular bombardment sites, low penetration depths, and sporadic editing efficiencies (Ahmar et al., 2021). Current genetic engineering methods also typically target immature, undifferentiated tissues (callus or meristems) and therefore require costly and laborious tissue culture protocols to generate progeny (Cunningham et al., 2018). Transient transformation of CRISPR-Cas reagents is preferred over stable integration due to fewer regulatory restrictions, shorter breeding cycles, and fewer unintended offtarget effects (Liang et al., 2017). This remains challenging due to the large cassette, high charge density, and low ionic stability of Cas9 protein, which needs to traverse through the protective plant cell wall. An alternative is first to introduce Cas9 ribonucleoproteins into wall-less protoplasts, but this requires tissue regeneration, which is challenging to use across species, especially for recalcitrant species (Mao et al., 2019). Consequently, an unmet need remains to devise an effective, low-cost, and universal strategy to deliver gene-editing cargo into plant cells.

\section{NANOTECHNOLOGY TO ADDRESS CHALLEGES FOR PLANT GENE EDITING}

Multiple proof-of-principle studies over the past few years have shown that nanoparticles, in particular carbon nanotubes (CNTs), can be used to deliver nucleic acid-based cargoes to plant species and tissues efficiently in an almost speciesindependent manner (Demirer et al., 2019; Kwak et al., 2019). Owing to the small size of nanoparticles (typically defined as those with dimensions of less than $500 \mathrm{~nm}$ ), they have been shown to transport past the plant cell wall and cellular membranes to deliver genetic cargo and to detect biomolecules (Lew et al., 2018; Lew et al., 2020a; Lew et al., 2020b; Lew et al., 2021). Transient expression of exogenous DNA in the chloroplasts and nucleus was demonstrated recently by leaf infiltration of cationic CNTs in mature arugula, spinach, wheat, and cotton, among others (Demirer et al., 2019; Kwak et al., 2019). The comprehensive molecular toolbox of surface chemistry enables facile functionalization of nanoparticles, potentially allowing the conjugation of CRISPR RNA-protein complexes to be protected from degradation, delivered to targeted cellular regions, and finally cleaved from the carrier in a controlled fashion, as has been demonstrated in mammalian systems (Deng et al., 2020; Wei et al., 2020). Direct penetration of pollen surface apertures for transformation could circumvent the need for regeneration from tissue culture, allowing direct production of edited offspring as demonstrated recently with single-walled CNTs (Lew et al., 2020c). Tissue culture and regeneration burden can also be reduced by targeting shoot apical meristem, which are usually inaccessible behind multiple tissue layers and require large penetration depths (Demirer et al., 2021). Layer-by-layer assembly on surface functionalized nanoparticles (Richardson et al., 2016) can potentially stagger Cas9 expression and sgRNA release for maximum transformation efficiency. Loading a whole array of nucleic acids or proteins on the same nanocarrier could simultaneously express multiple regulatory players in a controlled spatiotemporal fashion. This type of gene stacking is a promising approach for improving a complex desirable trait where multiple pathways need to be simultaneously perturbed but is challenging to achieve as genes need to be expressed in a targeted temporal manner which otherwise could lead to undesired pleiotropic effects (Vanhaeren et al., 2016).

\section{PLANT CELLULAR BARRIERS FOR TARGETED NANOPARTICLE DELIVERY}

While there have been successful laboratory demonstrations for nanoparticle trafficking into plant cells in recent years, the general mechanism of nanoparticle transport past the cell wall and the plasma membrane into various subcellular organelles remains unclear (Lew et al., 2020d). This lack of knowledge of nanoparticle interactions with plant cellular membranes has significantly hampered the development of reliable nanoscale tools to deliver gene-editing cargo (Lew et al., 2020a). For example, gene editing reagents can be accurately targeted to the mitochondria to engineer development and stress tolerance (Liberatore et al., 2016). Physical models explaining the distribution of nanoparticles within plant cells have been proposed, but how conjugation of biomolecular cargo alters nanoparticle localization remains understudied (Lew et al., 2018). Spatial control over the nanoparticle subcellular localization will uncover the potential utility of nanoparticles in organelle genome editing and metabolic engineering (Figure 2A) (Saminathan et al., 2021). Previously peptide biorecognition motif conjugations have been used to deliver plasmid DNA into chloroplast and mitochondria of intact plants (Yoshizumi et al., 2018; Thagun et al., 2019), but their use for targeting CRISPR Cas reagents remains unexplored. This is primarily due to the sub-efficient delivery of guide RNA and Cas9 into organelles which can be addressed by the available diversity of functionalization chemistries for peptide-nanoparticle conjugates. Leveraging endogenous receptor-ligand interactions can further allow targeting of loaded nanoparticles to specific cell types or organs in intact plants, as shown recently for C. elegans (Chakraborty et al., 2021). 

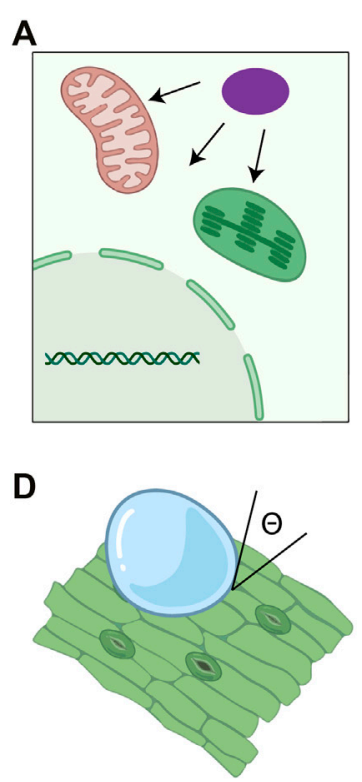

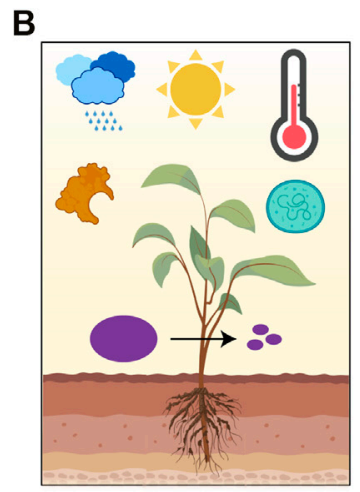

C

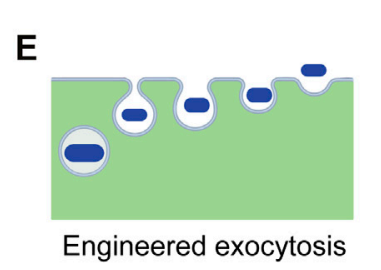

F
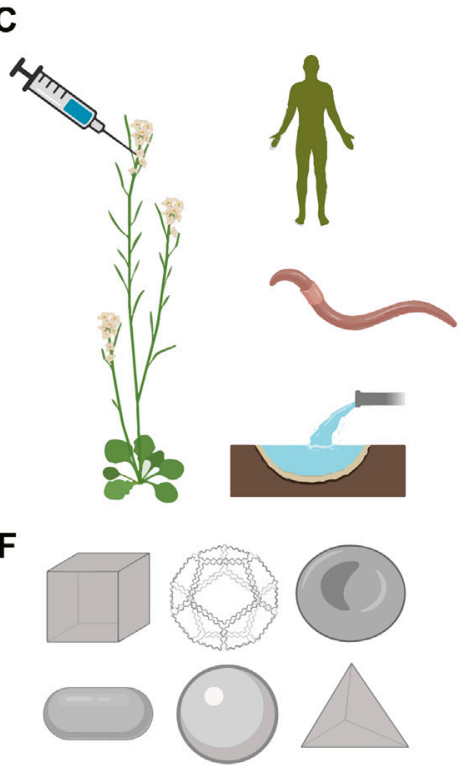

FIGURE 2 | (A) Targeting cargo, such as CRISPR reagents (purple), to organelles (green: chloroplast, red: mitochondria, and grey: nucleus) in plants allows the independent genetic engineering of these structures in their native context. (B) Nanoparticle design framework should account for the field conditions the cargo (purple) would be exposed to, such as sunlight, extreme temperatures, moisture, nucleases, and microbes. (C) Lifecycle assessment of plant nanoparticle gene editing effects on humans, soil biome, and nearby water ecosystems should be investigated. (D) Formulation optimization of the nanoparticle suspensiontomaximize wetting (represented by contact angle of suspension with leaf, $\Theta$ ) without compromising photosynthesis. (E) Exocytosis pathways of nanoparticles from plant cells and their engineering remains unclear. (F) Nanofabrication techniques such as DNA origami can be used to study the effects of nanoparticle size, shape, and functionalization on biodistribution and editing efficiencies (Created with BioRender.com).

The delivery barriers can be overcome by rationally manipulating nanoparticle design parameters such as size, shape, stiffness, and surface charge. In mammalian cells, nanoparticles averaging $\sim 100 \mathrm{~nm}$ generally undergo longlasting circulation (Blanco et al., 2015), while nanoparticles with diameters $<5 \mathrm{~nm}$ undergo rapid renal clearance and are eliminated from the body (Choi et al., 2007). Similarly, geometrical features such as particle orientation, aspect ratio, and curvature affect nanoparticle phagocytosis's kinetics, leading to the exploration of simple shapes such as cylindrical, ellipsoidal, and discoidal for cancer therapeutics (Dasgupta et al., 2014; Vácha et al., 2011; Champion and Mitragotri, 2006). The switchable surface charge has also been explored in mammalian systems to maximize the cellular uptake of nanoparticles (Yuan et al., 2012) since the negative charge promotes longer circulation times by decreasing nonspecific uptake (Yamamoto et al., 2001) while positive charge prevents cargo degradation within the endosomal compartment (Nel et al., 2009). Mechanical properties such as tensile strength and deformability also promote longer circulation and accumulation (Zhang et al., 2012). Similar counterpart explorations are necessary for plant cells since they differ from mammalian cells in several ways-additional cell wall barrier, porous cuticle, and the diversity of epidermal leaf features such as stomata and trichomes. Such optimization at the nanoparticle design stage will significantly reduce runoff losses to agricultural soils, associated environmental risks (increased exposure or change in exposure routes), and resource intensity (embodied water, energy, and emissions from upstream processing) while increasing genetic transformation efficiencies (Figures 2B,C) (Xi et al., 2021). However, conventional nanofabrication techniques do not allow the exploration of a more diverse shape and size design space. DNA origami is an especially promising branch of nanoparticles that utilizes Watson-Crick base pairing to enable high-yield and monodisperse synthesis of virtually any arbitrary structures with user-defined periodicity, asymmetry, or curvatures (Dietz et al., 2009; Han et al., 2011; Benson et al., 2015). Unique sequence and positions of staples allow nanometer-resolution addressability meaning functional moieties (including proteins and aptamers) can be sitespecifically placed at desired locations on the nanoparticle structure, which provides unmatched control of nanoparticlecell membrane interactions and uptake pathways (Bastings et al., 2018; Dey et al., 2021). The stoichiometry of cargo to scaffold molecules can be modulated to limit the wasteful runoffs and enable exceptional control over CRISPR reagent dosage per individual nanoparticle ( $\mathrm{Hu}$ et al., 2018). This is accompanied by the ability of these structures to resist enzymatic degradation (Mei et al., 2011), have minimal off-target effects (Lee et al., 2012), and release payloads in response to specific cues with spatial and temporal precision (Li et al., 2018). Many ever-expanding DNAmodification chemistries allow an unparalleled toolkit to explore the opportunity space of physical and chemical properties, (Yang et al., 2015; Stephanopoulos, 2019) thus presenting a versatile platform to investigate nanostructures' structure-property relationships for precision gene editing. 


\section{NANOPARTICLE-ENABLED PLANT GENE EDITING AT SCALE}

Plant gene editing at scale remains challenging to realize even in the presence of multiple successful studies showing the use of nanoparticles to deliver a variety of cargoes into intact plants. Most conventional gene-editing methods require regeneration from tissue culture, which remains a significant bottleneck in scaling (Zhang et al., 2020). The requirements for hormones and growth media are not defined for most species for differentiating somatic cells during tissue culture. Further, the cell passaging during culture is mutagenic and requires several months to years (Nasti and Voytas, 2021). Infiltration of nanoparticles into leaves is also currently limited by the obligatory use of puncturing and pressurizing (Demirer et al., 2019). With this method, the ratio of nanoparticles entering the plant to the applied amount is often overlooked, and it remains unclear why specific formulations demonstrate better uptake into the plant systems than the others (Su et al., 2019). Additionally, such a method is not amenable for large-scale applications outside the laboratory. An alternative way to introduce CRISPR cargo is through an aerosol-mediated foliar spray, where gene editing can be performed by spraying carbon nanoparticles similar to fertilizer application. This is especially promising for non-commercial applications such as genomewide organismal screens in a controlled laboratory setting. Further improvements will require careful optimization of the nanoparticle suspension formulations to increase wettability with leaf surface (smaller contact angle between leaf-suspension interface) and retention on leaf surface under field environment (sunlight, microbes, temperature, $\mathrm{pH}$, nucleases, organic matter, and rain) since leaves are the standard uptake route for nanoparticles in plants. This would also increase the intrinsic nanoparticle activity, i.e., observed transformation efficiencies normalized by the exposed surface area and loaded reagent amount.

Interestingly a wide variety of contact angles have been reported for leaves of different plant species with water (Wang et al., 2015; Kwak et al., 2017) implying that the variations in leaf morphological features may dictate the generalizability of using nanoparticle mediate gene editing across different plant species (Figure 2D). The leaf wettability depends on several factors, including the wax content and structure, trichome density, stomatal aperture and density, leaf water content, and shape of epidermal cells (Wang et al., 2015). Very high wettability of a suspension towards leaves may lead to a significant reduction in photosynthesis rates due to 10,000 fold difference in diffusion rates of carbon dioxide in water than air (Hanba et al., 2004). Surfactants can lower the surface tension of the nanoparticle formulations to increase adhesion and spread on the leaf surface but may denature the attached cargo proteins. Such surfactant molecules are also known to penetrate the cuticle and increase their conductance, increasing the nanoparticle uptake rates (Räsch et al., 2018). This presents a unique opportunity to characterize different plant species and optimize design formulations for maximal intrinsic nanoparticle activity without affecting plant growth rates. A few reports have shown promising results, which stress the importance of unexplored design space of wettability and sustained retention of nanoparticles. Layered double hydroxide clay nanosheets can protect dsRNA cargo from RNase treatment while adhering to the leaf surface even after vigorous rinsing and providing sustained release (Mitter et al., 2017). Deployment of nanoparticles for atscale plant gene editing is also limited by the technical issues of mass production, such as scalability, batch-batch variability, and reproducible performance under different environmental conditions.

\section{ENVIRONMENTAL AND REGULATORY CONSIDERATIONS FOR NANOPARTICLE-ENABLED PLANT GENE EDITING}

Classical genetically modified (GMO) crops are subjected to stringent regulatory frameworks due to the insertion of foreign DNA into the plant genome. In contrast to traditional transgenic approaches, nanoparticle-mediated delivery may enable transgene-free genome-edited crops via transient expression of CRISPR reagents. This could potentially circumvent GMO labeling in many countries and substantially lower the cost of regulatory processes associated with genome-edited crops, thus encouraging innovation, affordable access, and commercialization of these crops. However, the regulatory landscapes concerning plants modified with gene-editing technologies are different in various regions of the world. While the European Court of Justice's ruling to regulate genome-edited crops the same way as conventional GMO could stifle progress in plant genome editing (Zaidi et al., 2019), the rulings by United States and Japan to relax the regulations for genome-edited crops signaled a positive atmosphere which could lead to less restrictive regulatory purviews worldwide (Waltz, 2018).

The knowledge gaps in the understanding of the ecotoxicity, exposure pathways, and lifecycle impact assessments of nanoparticles may make it challenging to navigate the environmental and safety reviews by the regulatory agencies. This is further accompanied by other political, legal, consumer acceptance, economic, business, and ethical challenges about the use of nanoparticles (Yang and Duncan, 2021). Therefore, the question of the safe use of nanoparticles for plant gene editing for non-laboratory settings remains unexplored and will have to be reviewed by the public health agencies, consumers and manufacturers after considering environmental, performance, and economic trade-offs. This will add complexity to the already uncertain regulatory framework surrounding genomeedited crops. Such decision-making can be informed by sentinel field data collection at multiple test sites, though this will require dedicated long-term funding opportunities.

Nanotechnology still constitutes a novel tool to probe the fundamental biology of plants in plant science research by enabling genetic perturbations in a species-independent and efficient manner. While there remains a lack of comprehensive data on their effect on plant transcriptome and metabolic 
network, it can be addressed by the advent of next-generation omics technologies (such as genomics, transcriptomics, proteomics, and metabolomics), which can capture the molecule-level alterations introduced by these nanoparticles. Such information can then lead to a more advanced design of nanoparticles which may facilitate their rapid degradation, exocytosis, and excretion from plants after genetic cargo delivery, reducing toxicity or contamination concerns (Figures 2E,F). For example, peptide and chemical functionalization of nanoparticles has been used to regulate their exocytosis in mammalian cells (Bartczak et al., 2012; Oh and Park, 2014; Kim et al., 2015; Gravely et al., 2019), and similar strategies may be pursued to regulate nanoparticle fate in the plant cellular environment.

\section{CONCLUSION}

Nanoparticle-enabled gene-editing techniques have the potential to revolutionize agriculture owing to their ability to transform plants in a species-independent and nonintegrating manner. Facile surface chemistry allows versatile modification of nanoparticle physicochemical properties, allowing versatile functionalization to protect and safely deliver gene-editing cargoes into targeted compartments within plant cells. Barriers to the application of nanoparticles in agriculture include low transformation efficiency, in planta stability, lack of high-throughput delivery method, as well as understudied fate and exposure of these nanoparticles in the environment. In order to fully harness the potential of nanobiotechnology in agriculture, not

\section{REFERENCES}

Ahmar, S., King, C., Burgess, R. A., Bakare, A. A., Shittu, F., Salako, J., et al. (2021). Advantage of Nanotechnology-Based Genome Editing System and its Application in Crop Improvement. Front. Plant Sci. 12, 943. doi:10.3389/ fpls.2021.663849

Bailey-Serres, J., Parker, J. E., Ainsworth, E. A., Oldroyd, G. E. D., and Schroeder, J. I. (2019). Genetic Strategies for Improving Crop Yields. Nature 575, 109-118. doi:10.1038/s41586-019-1679-0

Bartczak, D., Nitti, S., Millar, T. M., and Kanaras, A. G. (2012). Exocytosis of Peptide Functionalized Gold Nanoparticles in Endothelial Cells. Nanoscale 4, 4470-4472. doi:10.1039/c2nr31064c

Bastings, M. M. C., Anastassacos, F. M., Ponnuswamy, N., Leifer, F. G., Cuneo, G., Lin, C., et al. (2018). Modulation of the Cellular Uptake of DNA Origami through Control over Mass and Shape. Nano Lett. 18, 3557-3564. doi:10.1021/ acs.nanolett.8b00660

Benson, E., Mohammed, A., Gardell, J., Masich, S., Czeizler, E., Orponen, P., et al. (2015). DNA Rendering of Polyhedral Meshes at the Nanoscale. Nature 523 (523), 441-444. doi:10.1038/nature14586

Bharat, S. S., Li, S., Li, J., Yan, L., and Xia, L. (2020). Base Editing in Plants: Current Status and Challenges. Crop J. 8, 384-395. doi:10.1016/j.cj.2019.10.002

Blanco, E., Shen, H., and Ferrari, M. (2015). Principles of Nanoparticle Design for Overcoming Biological Barriers to Drug Delivery. Nat. Biotechnol. 33 (33), 941-951. doi:10.1038/nbt.3330

Boutrot, F., and Zipfel, C. (2017). Function, Discovery, and Exploitation of Plant Pattern Recognition Receptors for Broad-Spectrum Disease Resistance. Annu. Rev. Phytopathol. 55, 257-286. doi:10.1146/annurevphyto-080614-120106 only do more research opportunities need to be generated to ensure nanoparticles' efficacy and safety, but social acceptance and an environment facilitating nanoparticle use in plant biotechnology are also essential. Although the field is still in its infancy, the increasing acceptance and changing regulatory landscape supporting nanoparticle use in healthcare and nanomedicine may signal a promising framework that can be adapted to facilitate the adoption of nanoparticles in agriculture. Nano-enabled precision breeding is predicted to be a powerful weapon against poverty and hunger, and conducive regulatory landscapes and support mechanisms need to be provided to develop this promising field.

\section{DATA AVAILABILITY STATEMENT}

The original contributions presented in the study are included in the article/Supplementary Material, further inquiries can be directed to the corresponding author.

\section{AUTHOR CONTRIBUTIONS}

PS and TL designed the study, generated ideas for discussion and wrote the manuscript.

\section{FUNDING}

TL acknowledges the support by the Agency of Science, Technology and Research (A`STAR) Career Development Fund.

Chakraborty, K., Anees, P., Surana, S., Martin, S., Aburas, J., Moutel, S., et al. (2021). Tissue-specific Targeting of DNA Nanodevices in a Multicellular Living Organism. Elife 10. doi:10.7554/eLife.67830

Champion, J. A., and Mitragotri, S. (2006). Role of Target Geometry in Phagocytosis. Proc. Natl. Acad. Sci. 103, 4930-4934. doi:10.1073/pnas. 0600997103

Chen, K., Wang, Y., Zhang, R., Zhang, H., and Gao, C. (2019). CRISPR/Cas Genome Editing and Precision Plant Breeding in Agriculture. Annu. Rev. Plant Biol. 70, 667-697. doi:10.1146/annurev-arplant-050718-100049

Choi, H. S., Liu, W., Misra, P., Tanaka, E., Zimmer, J. P., Itty Ipe, B., et al. (2007). Renal Clearance of Quantum Dots. Nat. Biotechnol. 25 (25), 1165-1170. doi:10. $1038 /$ nbt1340

Christian, M., Cermak, T., Doyle, E. L., Schmidt, C., Zhang, F., Hummel, A., et al. (2010). Targeting DNA Double-Strand Breaks with TAL Effector Nucleases. Genetics 186, 757-761. doi:10.1534/genetics.110.120717

Cunningham, F. J., Goh, N. S., Demirer, G. S., Matos, J. L., and Landry, M. P. (2018). Nanoparticle-Mediated Delivery towards Advancing Plant Genetic Engineering. Trends Biotechnol. 36, 882-897. doi:10.1016/j.tibtech.2018.03.009

Dar, M. H., Zaidi, N. W., Waza, S. A., Verulkar, S. B., Ahmed, T., Singh, P. K., et al. (2018). No Yield Penalty under Favorable Conditions Paving the Way for Successful Adoption of Flood Tolerant rice. Sci. Rep. 8 (8), 9245-9247. doi:10. 1038/s41598-018-27648-y

Dasgupta, S., Auth, T., and Gompper, G. (2014). Shape and Orientation Matter for the Cellular Uptake of Nonspherical Particles. Nano Lett. 14 (2), 687-693. doi:10.1021/nl403949h

Day, C. D., Lee, E., Kobayashi, J., Holappa, L. D., Albert, H., and Ow, D. W. (2000). Transgene Integration into the Same Chromosome Location Can Produce Alleles that Express at a Predictable Level, or Alleles that Are Differentially Silenced. Genes Dev. 14, 2869-2880. doi:10.1101/gad.849600 
Demirer, G. S., Zhang, H., Matos, J. L., Goh, N. S., Cunningham, F. J., Sung, Y., et al. (2019). High Aspect Ratio Nanomaterials Enable Delivery of Functional Genetic Material without DNA Integration in Mature Plants. Nat. Nanotechnol 14 (14), 456-464. doi:10.1038/s41565-019-0382-5

Demirer, G. S., Saliva, T., Jackson, C. T., Thomas, J., Ehrhardt, D., Rhee, S., et al. (2021). Nanotechnology to advance CRISPR-Cas Genetic Engineering of Plants. Nat. Nanotechnol. 163 (16), 243-250. doi:10.1038/s41565-021-00854-y

Deng, S., Li, X., Liu, S., Chen, J., Li, M., Chew, S. Y., et al. (2020). Codelivery of CRISPRCas9 and chlorin e6 for spatially controlled tumor-specific gene editing with synergistic drug effects. Sci. Adv. 6, eabb4005. doi:10.1126/sciadv.abb4005

Dey, S., Fan, C., Gothelf, K. V., Li, J., Lin, C., Liu, L., et al. (2021). DNA Origami. Nat. Rev. Methods Prim. 11 (1), 1-24. doi:10.1038/s43586-020-00009-8

Dietz, H., Douglas, S. M., and Shih, W. M. (2009). Folding DNA into Twisted and Curved Nanoscale Shapes. Science 325, 725-730. doi:10.1126/science.1174251

Dong, O. X., and Ronald, P. C. (2021). Targeted DNA Insertion in Plants. Proc. Natl. Acad. Sci. U S A. 118. doi:10.1073/pnas.2004834117

Duan, L., Ouyang, K., Xu, X., Xu, L., Wen, C., Zhou, X., et al. (2021). Nanoparticle Delivery of CRISPR/Cas9 for Genome Editing. Front. Genet. 12, 788. doi:10. 3389/fgene.2021.673286

Fausther-Bovendo, H., and Kobinger, G. (2021). Plant-made Vaccines and Therapeutics. Science 373, 740-741. doi:10.1126/science.abf5375

Gaillochet, C., Develtere, W., and Jacobs, T. B. (2020). CRISPR Screens in Plants: Approaches, Guidelines, and Future Prospects. Plant Cell 33 (4), 794-813. doi:10.1093/plcell/koab099

Gomez, M. A., Lin, Z. D., Moll, T., Chauhan, R. D., Hayden, L., Renninger, K., et al. (2019). Simultaneous CRISPR/Cas9-Mediated Editing of Cassava eIF4E Isoforms nCBP-1 and nCBP-2 Reduces Cassava Brown Streak Disease Symptom Severity and Incidence. Plant Biotechnol. J. 17, 421-434. doi:10.1111/pbi.12987

Gravely, M., Safaee, M. M., and Roxbury, D. (2019). Biomolecular Functionalization of a Nanomaterial to Control Stability and Retention within Live Cells. Nano Lett. 19, 6203-6212. doi:10.1021/acs.nanolett.9b02267

Han, D., Pal, S., Nangreave, J., Deng, Z., Liu, Y., and Yan, H. (2011). DNA Origami with Complex Curvatures in Three-Dimensional Space. Science 332, 342-346. doi:10.1126/science. 1202998

Hanba, Y. T., Kimura, K., and Kimura, K. (2004). Effect of Leaf Surface Wetness and Wettability on Photosynthesis in Bean and Pea. Plant Cel Environ. 27, 413-421. doi:10.1046/j.1365-3040.2004.01154.x

Hu, B., Wang, W., Ou, S., Tang, J., Li, H., Che, R., et al. (2015). Variation in NRT1.1B Contributes to Nitrate-Use Divergence between rice Subspecies. Nat. Genet. 47 (47), 834-838. doi:10.1038/ng.3337

Hu, Q., Li, H., Wang, L., Gu, H., and Fan, C. (2018). DNA NanotechnologyEnabled Drug Delivery Systems. Chem. Rev. 119, 6459-6506. doi:10.1021/acs. chemrev.7b00663

Jones, H. D. (2015). Regulatory Uncertainty over Genome Editing. Nat. Plants 1 (1), 14011-14013. doi:10.1038/nplants.2014.11

Kim, C., Tonga, G. Y., Yan, B., Kim, C. S., Kim, S. T., Park, M.-H., et al. (2015). Regulating Exocytosis of Nanoparticles via Host-Guest Chemistry. Org. Biomol. Chem. 13, 2474-2479. doi:10.1039/c4ob02433h

Klein, T. M., Arentzen, R., Lewis, P. A., and Fitzpatrick-McElligott, S. (1992). Transformation of Microbes, Plants and Animals by Particle Bombardment. Biotechnology (N Y) 10 (10), 286-291. doi:10.1038/nbt0392-286

Koller, T., Brunner, S., Herren, G., Sanchez-Martin, J., Hurni, S., Keller, B., et al. (2019). Field grown transgenic Pm3e wheat lines show powdery mildew resistance and no fitness costs associated with high transgene expression. Transgenic Res. 28, 9-20. doi:10.1007/s11248-018-0099-5

Koonin, E. V., Makarova, K. S., and Zhang, F. (2017). Diversity, Classification and Evolution of CRISPR-Cas Systems. Curr. Opin. Microbiol. 37, 67-78. doi:10. 1016/j.mib.2017.05.008

Kromdijk, J., Głowacka, K., Leonelli, L., Gabilly, S. T., Iwai, M., Niyogi, K. K., et al. (2016). Improving Photosynthesis and Crop Productivity by Accelerating Recovery from Photoprotection. Science 354, 857-861. doi:10.1126/science.aai8878

Kwak, S.-Y., Giraldo, J. P., Wong, M. H., Koman, V. B., Lew, T. T. S., Ell, J., et al. (2017). A Nanobionic Light-Emitting Plant. Nano Lett. 17, 7951-7961. doi:10. 1021/acs.nanolett.7b04369

Kwak, S. Y., Lew, T. T. S., Sweeney, C. J., Koman, V. B., Wong, M. H., BohmertTatarev, K., et al. (2019). Chloroplast-selective Gene Delivery and Expression in Planta Using Chitosan-Complexed Single-Walled Carbon Nanotube Carriers. Nat. Nanotechnol 14 (14), 447-455. doi:10.1038/s41565-019-0375-4
Lee, H., Lytton-Jean, A. K., Chen, Y., Love, K. T., Park, A. I., Karagiannis, E. D., et al. (2012). Molecularly Self-Assembled Nucleic Acid Nanoparticles for Targeted In Vivo siRNA Delivery. Nat. Nanotechnol. 7, 389-393. doi:10. 1038/nnano.2012.73

Lew, T. T. S., Park, M., Cui, J., and Strano, M. S. (2021). Plant Nanobionic Sensors for Arsenic Detection. Adv. Mater. 33, e2005683. doi:10.1002/adma.202005683

Lew, T. T. S., Sarojam, R., Jang, I. C., Park, B. S., Naqvi, N. I., Wong, M. H., et al. (2020). Species-independent Analytical Tools for Next-Generation Agriculture. Nat. Plants 6 (6), 1408-1417. doi:10.1038/s41477-020-00808-7

Lew, T. T. S., Koman, V. B., Gordiichuk, P., Park, M., and Strano, M. S. (2020). The Emergence of Plant Nanobionics and Living Plants as Technology. Adv. Mater. Technol. 5, 1900657. doi:10.1002/admt.201900657

Lew, T. T. S., Koman, V. B., Silmore, K. S., Seo, J. S., Gordiichuk, P., Kwak, S.-Y., et al. (2020). Real-time Detection of Wound-Induced $\mathrm{H} 2 \mathrm{O} 2$ Signalling Waves in Plants with Optical Nanosensors. Nat. Plants 6, 404-415. doi:10.1038/ s41477-020-0632-4

Lew, T. T. S., Park, M., Wang, Y., Gordiichuk, P., Yeap, W.-C., Mohd Rais, S. K., et al. (2020). Nanocarriers for Transgene Expression in Pollen as a Plant Biotechnology Tool. ACS Mater. Lett. 2, 1057-1066. doi:10.1021/acsmaterialslett.0c00247

Lew, T. T. S., Wong, M. H., Kwak, S.-Y., Sinclair, R., Koman, V. B., and Strano, M. S. (2018). Rational Design Principles for the Transport and Subcellular Distribution of Nanomaterials into Plant Protoplasts. Small 14, 1802086. doi:10.1002/smll.201802086

Li, M., Li, X., Zhou, Z., Wu, P., Fang, M., Pan, X., et al. (2016). Reassessment of the Four Yield-Related Genes Gn1a, DEP1, GS3, and IPA1 in rice Using a CRISPR/ Cas9 System. Front. Plant Sci. 7, 377. doi:10.3389/fpls.2016.00377

Li, S., Jiang, Q., Liu, S., Zhang, Y., Tian, Y., Song, C., et al. (2018). A DNA Nanorobot Functions as a Cancer Therapeutic in Response to a Molecular Trigger In Vivo. Nat. Biotechnol. 36 (36), 258-264. doi:10.1038/nbt.4071

Liang, Z., Chen, K., Li, T., Zhang, Y., Wang, Y., Zhao, Q., et al. (2017). Efficient DNA-free Genome Editing of Bread Wheat Using CRISPR/Cas9 Ribonucleoprotein Complexes. Nat. Commun. 8 (8), 14261-14265. doi:10. 1038/ncomms14261

Liberatore, K. L., Dukowic-Schulze, S., Miller, M. E., Chen, C., and Kianian, S. F. (2016). The Role of Mitochondria in Plant Development and Stress Tolerance. Free Radic. Biol. Med. 100, 238-256. doi:10.1016/j.freeradbiomed.2016.03.033

Ma, X., Zhang, X., Liu, H., and Li, Z. (2020). Highly Efficient DNA-free Plant Genome Editing Using Virally Delivered CRISPR-Cas9. Nat. Plants 6 (6), 773-779. doi:10.1038/s41477-020-0704-5

Mali, P., Yang, L., Esvelt, K. M., Aach, J., Guell, M., DiCarlo, J. E., et al. (2013). RNA-guided Human Genome Engineering via Cas9. Science 339, 823-826. doi:10.1126/science. 1232033

Mao, Y., Botella, J. R., Liu, Y., and Zhu, J.-K. (2019). Gene Editing in Plants: Progress and Challenges. Natl. Sci. Rev. 6, 421-437. doi:10.1093/nsr/nwz005

Mei, Q., Wei, X., Su, F., Liu, Y., Youngbull, C., Johnson, R., et al. (2011). Stability of DNA Origami Nanoarrays in Cell Lysate. Nano Lett. 11, 1477-1482. doi:10. 1021/nl1040836

Meyer, P. (1995). Understanding and Controlling Transgene Expression. Trends Biotechnol. 13, 332-337. doi:10.1016/S0167-7799(00)88977-5

Miller, K., Eggenberger, A. L., Lee, K., Liu, F., Kang, M., Drent, M., et al. (2021). An Improved Biolistic Delivery and Analysis Method for Evaluation of DNA and CRISPR-Cas Delivery Efficacy in Plant Tissue. Sci. Rep. 111 (11), 1-11. doi:10. 1038/s41598-021-86549-9

Mitter, N., Worrall, E. A., Robinson, K. E., Li, P., Jain, R. G., Taochy, C., et al. (2017). Clay Nanosheets for Topical Delivery of RNAi for Sustained protection against Plant Viruses. Nat. Plants 3 (3), 16207-16210. doi:10.1038/nplants. 2016.207

Mumm, R. H., and Walters\&, D. S. (2001). Quality Control in the Development of Transgenic Crop Seed Products. Crop Sci. 41, 1381-1389. doi:10.2135/ cropsci2001.4151381x

Nasti, R. A., and Voytas, D. F. (2021). Attaining the Promise of Plant Gene Editing at Scale. Proc. Natl. Acad. Sci. U S A. 118. doi:10.1073/pnas.2004846117

Nel, A. E., Mädler, L., Velegol, D., Xia, T., Hoek, E. M., Somasundaran, P., et al. (2009). Understanding Biophysicochemical Interactions at the Nano-Bio Interface. Nat. Mater. 8 (8), 543-557. doi:10.1038/nmat2442

Oh, N., and Park, J.-H. (2014). Surface Chemistry of Gold Nanoparticles Mediates Their Exocytosis in Macrophages. ACS Nano 8, 6232-6241. doi:10.1021/ nn501668a 
Oliva, R., Ji, C., Atienza-Grande, G., Huguet-Tapia, J. C., Perez-Quintero, A., Li, T., et al. (2019). Broad-spectrum Resistance to Bacterial Blight in rice Using Genome Editing. Nat. Biotechnol. 37 (37), 1344-1350. doi:10.1038/s41587019-0267-z

Padmaja, G. (1995). Cyanide Detoxification in Cassava for Food and Feed Uses. Crit. Rev. Food Sci. Nutr. 35, 299-339. doi:10.1080/10408399509527703

Räsch, A., Hunsche, M., Mail, M., Burkhardt, J., Noga, G., and Pariyar, S. (2018). Agricultural Adjuvants May Impair Leaf Transpiration and Photosynthetic Activity. Plant Physiol. Biochem. 132, 229-237. doi:10.1016/j.plaphy.2018.08.042

Ray, D. K., Mueller, N. D., West, P. C., and Foley, J. A. (2013). Yield Trends Are Insufficient to Double Global Crop Production by 2050. PLoS One 8, e66428. doi:10.1371/journal.pone.0066428

Richardson, J. J., Cui, J., Björnmalm, M., Braunger, J. A., Ejima, H., and Caruso, F. (2016). Innovation in Layer-By-Layer Assembly. Chem. Rev. 116, 14828-14867. doi:10.1021/acs.chemrev.6b00627

Saminathan, A., Zajac, M., Anees, P., and Krishnan, Y. (2021). Organelle-level Precision with Next-Generation Targeting Technologies. Nat. Rev. Mater. 2021, 1-17. doi:10.1038/s41578-021-00396-8

Sandhya, D., Jogam, P., Allini, V. R., Abbagani, S., and Alok, A. (2020). The Present and Potential Future Methods for Delivering CRISPR/Cas9 Components in Plants. J. Genet. Eng. Biotechnol. 18, 25. doi:10.1186/s43141-020-00036-8

Sb, G. (2017). Integration of Agrobacterium T-DNA into the Plant Genome. Annu. Rev. Genet. 51, 195-217.

Schiml, S., Fauser, F., and Puchta, H. (2014). The CRISPR/Cas System Can Be Used as Nuclease Forin Plantagene Targeting and as Paired Nickases for Directed Mutagenesis in Arabidopsis Resulting in Heritable Progeny. Plant J. 80, 1139-1150. doi:10.1111/tpj.12704

Shimatani, Z., Kashojiya, S., Takayama, M., Terada, R., Arazoe, T., Ishii, H., et al. (2017). Targeted Base Editing in rice and Tomato Using a CRISPR-Cas9 Cytidine Deaminase Fusion. Nat. Biotechnol. 35 (35), 441-443. doi:10.1038/nbt.3833

Stephanopoulos, N. (2019). Peptide-Oligonucleotide Hybrid Molecules for Bioactive Nanomaterials. Bioconjug. Chem. 30, 1915-1922. doi:10.1021/acs. bioconjchem.9b00259

Su, Y., Ashworth, V., Kim, C., Adeleye, A. S., Rolshausen, P., Roper, C., et al. (2019). Delivery, Uptake, Fate, and Transport of Engineered Nanoparticles in Plants: a Critical Review and Data Analysis. Environ. Sci. Nano 6, 2311-2331. doi:10. 1039/c9en00461k

Thagun, C., Chuah, J. A., and Numata, K. (2019). Targeted Gene Delivery into Various Plastids Mediated by Clustered Cell-Penetrating and ChloroplastTargeting Peptides. Adv. Sci. 6, 1902064. doi:10.1002/advs.201902064

Tian, S., Jiang, L., Cui, X., Zhang, J., Guo, S., Li, M., et al. (2018). Engineering HerbicideResistant Watermelon Variety through CRISPR/Cas9-mediated Base-Editing. Plant Cel Rep 37 (37), 1353-1356. doi:10.1007/s00299-018-2299-0

Tilman, D., Balzer, C., Hill, J., and Befort, B. L. (2011). Global Food Demand and the Sustainable Intensification of Agriculture. Proc. Natl. Acad. Sci. 108, 20260-20264. doi:10.1073/pnas.1116437108

Vácha, R., Martinez-Veracoechea, F. J., and Frenkel, D. (2011). Receptor-mediated Endocytosis of Nanoparticles of Various Shapes. Nano Lett. 11, 5391-5395. doi:10.1021/nl2030213

Vanhaeren, H., Inzé, D., and Gonzalez, N. (2016). Plant Growth beyond Limits. Trends Plant Sci. 21, 102-109. doi:10.1016/j.tplants.2015.11.012

Voesenek, L. A. C. J., and Bailey-Serres, J. (2015). Flood Adaptive Traits and Processes: an Overview. New Phytol. 206, 57-73. doi:10.1111/nph.13209

Waltz, E. (2018). With a Free Pass, CRISPR-Edited Plants Reach Market in Record Time. Nat. Biotechnol. 36, 6-7. doi:10.1038/nbt0118-6b

Wang, H., Shi, H., and Wang, Y. (2015). The Wetting of Leaf Surfaces and its Ecological Significances. Wetting and Wettability 1, 1. doi:10.5772/61205

Wang, Y., Cheng, X., Shan, Q., Zhang, Y., Liu, J., Gao, C., et al. (2014). Simultaneous Editing of Three Homoeoalleles in Hexaploid Bread Wheat Confers Heritable Resistance to Powdery Mildew. Nat. Biotechnol. 32 (32), 947-951. doi:10.1038/nbt.2969

Wei, T., Cheng, Q., Min, Y. L., Olson, E. N., and Siegwart, D. J. (2020). Systemic Nanoparticle Delivery of CRISPR-Cas9 Ribonucleoproteins for Effective Tissue Specific Genome Editing. Nat. Commun. 11 (11), 3232. doi:10.1038/s41467020-17029-3

Wright, D. A., Townsend, J. A., Winfrey, R. J., Irwin, P. A., Rajagopal, J., Lonosky, P. M., et al. (2005). High-frequency Homologous Recombination in Plants Mediated by Zinc-finger Nucleases. Plant J. 44, 693-705. doi:10.1111/j.1365-313x.2005.02551.x
Xi, L., Zhang, M., Zhang, L., Lew, T. T. S., and Lam, Y. M. (2021). Novel Materials for Urban Farming. Adv. Mater. 1, 2105009. doi:10.1002/adma.202105009

Yamamoto, Y., Nagasaki, Y., Kato, Y., Sugiyama, Y., and Kataoka, K. (2001). Longcirculating Poly(ethylene Glycol)-Poly(d,l-Lactide) Block Copolymer Micelles with Modulated Surface Charge. J. Controlled Release 77, 27-38. doi:10.1016/ s0168-3659(01)00451-5

Yang, T., and Duncan, T. V. (2021). Challenges and Potential Solutions for Nanosensors Intended for Use with Foods. Nat. Nanotechnol. 163 (16), 251-265. doi:10.1038/s41565-021-00867-7

Yang, Y. R., Liu, Y., and Yan, H. (2015). DNA Nanostructures as Programmable Biomolecular Scaffolds. Bioconjug. Chem. 26, 1381-1395. doi:10.1021/acs. bioconjchem.5b00194

Yoshizumi, T., Oikawa, K., Chuah, J.-A., Kodama, Y., and Numata, K. (2018). Selective Gene Delivery for Integrating Exogenous DNA into Plastid and Mitochondrial Genomes Using Peptide-DNA Complexes. Biomacromolecules 19, 1582-1591. doi:10.1021/acs.biomac.8b00323

Yu, Q., Huang, H., Chen, J., Hu, X., Chen, Y., Xie, J., et al. (2021). RNA Demethylation Increases the Yield and Biomass of rice and Potato Plants in Field Trials. Nat. Biotechnol. 2021, 1-8. doi:10.1038/s41587-021-00982-9

Yuan, Y.-Y., Mao, C.-Q., Du, X.-J., Du, J.-Z., Wang, F., and Wang, J. (2012). Surface Charge Switchable Nanoparticles Based on Zwitterionic Polymer for Enhanced Drug Delivery to Tumor. Adv. Mater. 24, 5476-5480. doi:10. 1002/adma.201202296

Zaidi, S. S.-E.-A., Vanderschuren, H., Qaim, M., Mahfouz, M. M., Kohli, A., Mansoor, S., et al. (2019). New Plant Breeding Technologies for Food Security. Science 363, 1390-1391. doi:10.1126/science.aav6316

Zhang, L., Cao, Z., Li, Y., Ella-Menye, J. R., Bai, T., Jiang, S., et al. (2012). Softer Zwitterionic Nanogels for Longer Circulation and Lower Splenic Accumulation. ACS Nano 6, 6681-6686. doi:10.1021/nn301159a

Zhang, R., Liu, J., Chai, Z., Chen, S., Bai, Y., Zong, Y., et al. (2019). Generation of Herbicide Tolerance Traits and a New Selectable Marker in Wheat Using Base Editing. Nat. Plants 5 (5), 480-485. doi:10.1038/s41477-019-0405-0

Zhang, Y., Malzahn, A. A., Sretenovic, S., and Qi, Y. (2019). The Emerging and Uncultivated Potential of CRISPR Technology in Plant Science. Nat. Plants 5 (5), 778-794. doi:10.1038/s41477-019-0461-5

Zhang, Y., Pribil, M., Palmgren, M., and Gao, C. (2020). A CRISPR Way for Accelerating Improvement of Food Crops. Nat. Food 14 (1), 200-205. doi:10. 1038/s43016-020-0051-8

Zhang, Z., Hua, L., Gupta, A., Tricoli, D., Edwards, K. J., Yang, B., et al. (2019). Development of an Agrobacterium -delivered CRISPR/Cas9 System for Wheat Genome Editing. Plant Biotechnol. J. 17, 1623-1635. doi:10.1111/pbi.13088

Zhao, K., Tung, C. W., Eizenga, G. C., Wright, M. H., Ali, M. L., Price, A. H., et al. (2011). Genome-wide Association Mapping Reveals a Rich Genetic Architecture of Complex Traits in Oryza Sativa. Nat. Commun. 2 (2), 467. doi:10.1038/ncomms1467

Zhao, X., Meng, Z., Wang, Y., Chen, W., Sun, C., Cui, B., et al. (2017). Pollen Magnetofection for Genetic Modification with Magnetic Nanoparticles as Gene Carriers. Nat. Plants 3, 956-964. doi:10.1038/s41477-017-0063-Z

Zhao, Y., Zhang, C., Liu, W., Gao, W., Liu, C., Song, G., et al. (2016). An Alternative Strategy for Targeted Gene Replacement in Plants Using a Dual-sgRNA/Cas9 Design. Sci. Rep. 6 (6), 23890. doi:10.1038/srep23890

Conflict of Interest: The authors declare that the research was conducted in the absence of any commercial or financial relationships that could be construed as a potential conflict of interest.

Publisher's Note: All claims expressed in this article are solely those of the authors and do not necessarily represent those of their affiliated organizations, or those of the publisher, the editors and the reviewers. Any product that may be evaluated in this article, or claim that may be made by its manufacturer, is not guaranteed or endorsed by the publisher.

Copyright $\odot 2022$ Sharma and Lew. This is an open-access article distributed under the terms of the Creative Commons Attribution License (CC BY). The use, distribution or reproduction in other forums is permitted, provided the original author(s) and the copyright owner(s) are credited and that the original publication in this journal is cited, in accordance with accepted academic practice. No use, distribution or reproduction is permitted which does not comply with these terms. 